\title{
International Year of the Periodic Table from a Physical Chemistry Perspective Virtual Issue
}

\section{Helium}

Helium Accepts Back-Donation In Highly Polar Complexes: New Insights into the Weak Chemical Bond

Francesca Nunzi, ${ }^{*}$ Diego Cesario, Fernando Pirani, Leonardo Belpassi, Gernot Frenking, Felice Grandinett, Francesco Tarantelli*

J. Phys. Chem. Lett. 2017, 8, 14, 3334-3340.

https://doi.org/10.1021/acs.jpclett.7b01320

\section{Lithium}

Lithium Metal Anodes: Toward an Improved Understanding of Coupled Morphological, Electrochemical, and Mechanical Behavior

Kevin N. Wood, Malachi Noked, Neil P. Dasgupta*

ACS Energy Lett. 2017, 2, 3, 664-672.

https://doi.org/10.1021/acsenergylett.6b00650

\section{Boron}

Mechanistic Investigation of Thermal and Photoreactions between Boron and Silane Jiwon Moon, Heehyun Baek, Joonghan Kim*

J. Phys. Chem. A 2017, 121, 35, 6531-6537.

https://doi.org/10.1021/acs.jpca.7b03202

\section{Carbon}

CH4/CO2 Mixture Adsorption on a Characterized Activated Carbon

David Pino,* David Bessieres

J. Chem. Eng. Data 2017, 62, 4, 1475-1480.

https://doi.org/10.1021/acs.jced.6b01029

\section{Nitrogen}

The Abiotic Nitrogen Cycle 
Timothy A. Doane*

ACS Earth Space Chem. 2017, 1, 7, 411-421.

https://doi.org/10.1021/acsearthspacechem.7b00059

Theoretical Studies on the Energetic Salts of Substituted 3,3'-Amino-N,N'-azo-1,2,4-triazoles: The Role of Functional Groups

Xueli Zhang, Xuedong Gong*

J. Chem. Eng. Data 2015, 60, 10 2869-2878.

https://doi.org/10.1021/acs.jced.5b00257

\section{Oxygen}

No Photon Wasted: An Efficient and Selective Singlet Oxygen Photosensitizing Protein Michael Westberg, Mikkel Bregnhøj, Michael Etzerodt, Peter R. Ogilby*

J. Phys. Chem. B 2017, 121, 40, 9366-9371.

https://doi.org/10.1021/acs.jpcb.7b07831

Neon

NeON+: An Atom and a Molecule

Ryan C. Fortenberry,* Steven R. Gwaltney

ACS Earth Space Chem. 2018, 2, 5, 491-495.

https://doi.org/10.1021/acsearthspacechem.8b00019

\section{Sodium}

Accurate Binding of Sodium and Calcium to a POPC Bilayer by Effective Inclusion of Electronic Polarization

Josef Melcr, Hector Martinez-Seara, Ricky Nencini, Jiří Kolafa, Pavel Jungwirth, O. H. Samuli Ollila*

J. Phys. Chem. B 2018, 122, 16, 4546-4557.

https://doi.org/10.1021/acs.jpcb.7b12510

\section{Magnesium}

Thermostability of the PYL-PP2C Heterodimer Is Dependent on Magnesium: In Silico Insights into the Link between Heat Stress Response and Magnesium Deficiency in Plants 
Emel Timucin,* Osman Ugur Sezerman

J. Chem. Inf. Model. 2018, 58, 3, 661-672.

https://doi.org/10.1021/acs.jcim.7b00655

\section{Aluminum}

\section{Revisiting the Corrosion of the Aluminum Current Collector in Lithium-Ion Batteries}

Tianyuan Ma, Gui-Liang Xu, Yan Li, Li Wang, Xiangming He, Jianming Zheng, Jun Liu, Mark H.

Engelhard, Peter Zapol, Larry A. Curtiss, Jacob Jorne, ${ }^{*}$ Khalil Amine, Zonghai Chen*

J. Phys. Chem. Lett. 2017, 8, 5, 1072-1077.

https://doi.org/10.1021/acs.jpclett.6b02933

\section{Silicon}

\section{Efficient Silicon Metasurfaces for Visible Light}

Zhenpeng Zhou, OrcidJuntao Li, Rongbin Su, Beimeng Yao, Hanlin Fang, Kezheng Li, Lidan Zhou, Jin Liu, Daan Stellinga, Christopher P. Reardon, Thomas F. Krauss, Xuehua Wang*

ACS Photonics 2017, 4, 3, 544-551.

https://doi.org/10.1021/acsphotonics.6b00740

\section{Sulfur}

Hydrogen Sulfide as a Scavenger of Sulfur Atomic Cation

Ryan C. Fortenberry, *Tarek Trabelsi, Joseph S. Francisco

J. Phys. Chem. A 2018, 122, 22, 4983-4987.

https://doi.org/10.1021/acs.jpca.8b02923

\section{Argon}

Molecular Models for the Hydrogen Age: Hydrogen, Nitrogen, Oxygen, Argon, and Water Andreas Köster, Monika Thol, Jadran Vrabec*

J. Chem. Eng. Data 2018, 63, 2, 305-320.

https://doi.org/10.1021/acs.jced.7b00706

\section{Calcium}

Membrane Charging and Swelling upon Calcium Adsorption as Revealed by Phospholipid Nanodiscs 
Orion Shih, Yi-Qi Yeh, Kuei-Fen Liao, Chun-Jen Su, Pei-Hao Wu, Richard K. Heenan, Tsyr-Yan Yu,* U-Ser Jeng*

J. Phys. Chem. Lett. 2018, 9, 15, 4287-4293.

https://doi.org/10.1021/acs.jpclett.8b01651

\section{Cobalt}

Role of Transient Co-Subcarbonyls in Ostwald Ripening Sintering of Cobalt Supported on $\mathbf{y -}$ Alumina Surfaces

Werner Janse van Rensburg, Pieter van Helden,* Denzil J. Moodley, Michael Claeys, Melissa A. Petersen, Eric van Steen*

J. Phys. Chem. C 2017, 121, 31, 16739-16753.

https://doi.org/10.1021/acs.jpcc.7b01907

Nickel

Experimental Study and Mathematical Modeling of Self-Sustained Kinetic Oscillations in Catalytic Oxidation of Methane over Nickel

Elena A. Lashina, Vasily V. Kaichev, * Andrey A. Saraev, Zakhar S. Vinokurov, Nataliya A. Chumakova. Gennadii A. Chumakov, Valerii I. Bukhtiyarov

J. Phys. Chem. A 2017, 121, 37, 6874-6886.

https://doi.org/10.1021/acs.jpca.7b04525

\section{Copper}

Electro- and Photoreduction of Carbon Dioxide: The Twain Shall Meet at Copper Oxide/Copper Interfaces

C. Janáky, D. Hursán. B. Endrődi, W. Chanmanee, D. Roy, D. Liu, N. R. de Tacconi, B. H. Dennis, K. Rajeshwar*

ACS Energy Lett. 2016, 1, 2, 332-338.

https://doi.org/10.1021/acsenergylett.6b00078

Zinc

QM/MM Calculation of the Enzyme Catalytic Cycle Mechanism for Copper- and ZincContaining Superoxide Dismutase

Masami Lintuluoto, ${ }^{*}$ Chiaki Yamada, Juha M. Lintuluoto 
J. Phys. Chem. B 2017, 121, 30, 7235-7246.

https://doi.org/10.1021/acs.jpcb.7b03589

\section{Germanium}

Degenerate Four-Wave Mixing in a Multiresonant Germanium Nanodisk

Gustavo Grinblat,*Yi Li,* Michael P. Nielsen, Rupert F. Oulton, Stefan A. Maier

ACS Photonics 2017, 4, 9, 2144-2149.

https://doi.org/10.1021/acsphotonics.7b00631

\section{Selenium}

Three-Dimensional Hierarchical Graphene-CNT@Se: A Highly Efficient Freestanding Cathode for Li-Se Batteries

Jiarui He, Yuanfu Chen, *Weiqiang Lv, Kechun Wen, Pingjian Li, Zegao Wang, Wanli Zhang, Wu Qin, Weidong He*

ACS Energy Lett. 2016, 1, 1, 16-20.

https://doi.org/10.1021/acsenergylett.6b00015

\section{Bromine}

Modeling of Halogen-Protein Interactions in Co-Solvent Molecular Dynamics Simulations

Ying Yang, Amr H. Mahmoud, Markus A. Lill*

J. Chem. Inf. Model. 2019, 59, 1, 38-42.

https://doi.org/10.1021/acs.jcim.8b00806

\section{Zirconium}

Effects of Ligand Environment in Zr(IV) Assisted Peptide Hydrolysis

Tingting Zhang, Gaurav Sharma, Thomas J. Paul, Zachary Hoffmann, Rajeev Prabhakar*

J. Chem. Inf. Model. 2017, 57, 5, 1079-1088.

https://doi.org/10.1021/acs.jcim.6b00781

\section{Rhodium}

Adsorption and Activation of Water on Cuboctahedral Rhodium and Platinum Nanoparticles Andrey S. Bazhenov, Leon Lefferts, Karoliina Honkala* 
J. Phys. Chem. C 2017, 121, 8, 4324-4331.

https://doi.org/10.1021/acs.jpcc.6b11953

\section{Silver}

Hot Carrier Generation and Extraction of Plasmonic Alloy Nanoparticles

Marco Valenti, *Anirudh Venugopal, Daniel Tordera, Magnus P. Jonsson, George Biskos, Andreas Schmidt-Ott, Wilson A. Smith*

ACS Photonics 2017, 4, 5, 1146-1152.

https://doi.org/10.1021/acsphotonics.6b01048

\section{Lanthanum}

Improved Segmented All-Electron Relativistically Contracted Basis Sets for the Lanthanides

Daniel Aravena, Frank Neese,* Dimitrios A. Pantazis*

J. Chem. Theory Comput. 2016, 12, 3, 1148-1156.

https://doi.org/10.1021/acs.jctc.5b01048

\section{Rhenium}

Exploring the Mechanism of Ultrafast Intersystem Crossing in Rhenium(I) Carbonyl Bipyridine Halide Complexes: Key Vibrational Modes and Spin-Vibronic Quantum Dynamics

Yu Harabuchi, Julien Eng, Etienne Gindensperger, ${ }^{*}$ Tetsuya Taketsugu, Satoshi Maeda, ${ }^{*}$ Chantal Daniel*

J. Chem. Theory Comput. 2016, 12, 5 2335-2345.

https://doi.org/10.1021/acs.jctc.6b00080

\section{Osmium}

Metal-Metal Quintuple and Sextuple Bonding in Bent Dimetallocenes of the Third Row Transition Metals

Bing Xu, Qian-Shu Li* Yaoming Xie, R. Bruce King, * Henry F. Schaefer, III

J. Chem. Theory Comput. 2010, 6, 3, 735-746.

https://doi.org/10.1021/ct900564p

Iridium 
Monodisperse Iridium Clusters Protected by Phenylacetylene: Implication for Size-Dependent Evolution of Binding Sites

Hiroki Yamamoto, Prasenjit Maity, Ryo Takahata, Seiji Yamazoe, Kiichirou Koyasu, Wataru Kurashige, Yuichi Negishi, Tatsuya Tsukuda*

J. Phys. Chem. C 2017, 121, 20, 10936-10941.

https://doi.org/10.1021/acs.jpcc.6b12121

\section{Mercury}

Photomicrobial Visible Light-Induced Magnetic Mass Independent Fractionation of Mercury in a Marine Microalga

K. Kritee* Laura C. Motta, Joel D. Blum, Martin Tsz-Ki Tsui, John R. Reinfelder

ACS Earth Space Chem. 2018, 2, 5, 432-440.

https://doi.org/10.1021/acsearthspacechem.7b00056

\section{Lead}

Characterizing the Mechanisms of Lead Immobilization via Bioapatite and Various Clay Minerals

Zhen Li,* Lingyi Tang, Yangfan Zheng, Da Tian, Mu Su, Fan Zhang, Shuojia Ma, Shuijin Hu* ACS Earth Space Chem. 2017, 1, 3, 152-157.

https://doi.org/10.1021/acsearthspacechem.7b00016 